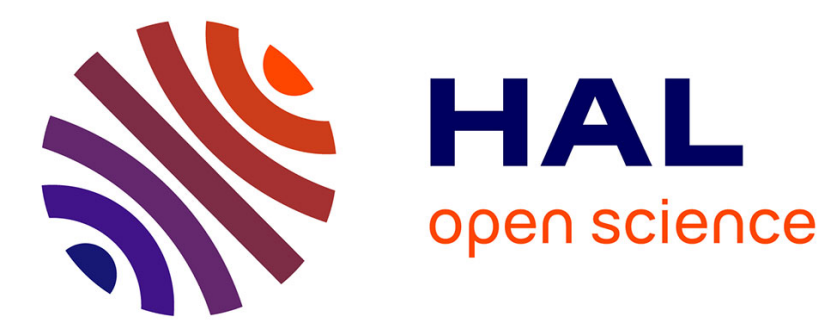

\title{
THz-wave sensing with two-photon spectroscopy of cold trapped $\mathrm{HD}+$
}

Florin Lucian Constantin

\section{To cite this version:}

Florin Lucian Constantin. THz-wave sensing with two-photon spectroscopy of cold trapped HD+. SPIE Photonex and Vacuum Expo 2020, Oct 2020, Coventry (on line), United Kingdom. pp.115780E, 10.1117/12.2583492 . hal-03431881

\section{HAL Id: hal-03431881 \\ https://hal.science/hal-03431881}

Submitted on 16 Nov 2021

HAL is a multi-disciplinary open access archive for the deposit and dissemination of scientific research documents, whether they are published or not. The documents may come from teaching and research institutions in France or abroad, or from public or private research centers.
L'archive ouverte pluridisciplinaire HAL, est destinée au dépôt et à la diffusion de documents scientifiques de niveau recherche, publiés ou non, émanant des établissements d'enseignement et de recherche français ou étrangers, des laboratoires publics ou privés.

$$
\text { Copyright }
$$




\title{
THz-wave sensing with two-photon spectroscopy of cold trapped HD ${ }^{+}$
}

\author{
F. L. Constantin*
}

Laboratoire PhLAM, CNRS UMR 8523, 59655 Villeneuve d'Ascq, France

\begin{abstract}
The comparison between precision spectroscopy measurements with cold trapped $\mathrm{HD}^{+}$ ions and accurate predictions of the molecular ion theory is exploited to characterize a $\mathrm{THz}$ electric field. A THz-wave off-resonantly coupled to the rotational transition at $1.3 \mathrm{THz}$ may be detected by measuring the lightshift induced on a Zeeman component of the two-photon rovibrational transition at 55.9 THz. A set of six lightshift measurements for two different orientations of the magnetic field in the ion trap can be converted into the amplitudes and the phases of the $\mathrm{THz}$ electric field components in a Cartesian laboratory reference frame.
\end{abstract}

Keywords: THz sensing, cold trapped ions, electrometry, two-photon spectroscopy, ac-Stark shift, Zeeman effect

*fl.constantin@univ-lille1.fr 


\section{INTRODUCTION}

Sensing of the electromagnetic fields has applications in geophysics, navigation, medical imaging and precision measurements. Atom-based detection of the microwave fields was exploited for SItraceable measurements, sub-wavelength resolution, accuracy, precision and long-term reproducible operation. Spectroscopy of Rydberg atoms, with photon shot noise limited sensitivity, allowed scalar detection of $\mu \mathrm{V} / \mathrm{cm}$ microwave electric fields ${ }^{1}$. This contribution proposes to characterize a $\mathrm{THz}$ electric field by comparing ab-initio predictions and experimental measurements of the lighshifts induced on a transition probed on cold trapped $\mathrm{HD}^{+}$ions. The hydrogen molecular ions are the simplest molecular systems for which the energies ${ }^{2,3}$ and the shifts in external fields ${ }^{4,5,6}$ were predicted accurately by ab-initio calculations. The transitions of the $\mathrm{HD}^{+}$ions in the mid-infrared and $\mathrm{THz}$ domain were addressed in radiofrequency traps with high resolution and accuracy, by exploiting the sympathetic cooling with laser-cooled $\mathrm{Be}^{+}$ions. The sub-Doppler spectroscopy techniques may allow significant improvements of the resolution and of the accuracy. The fundamental rotational transition of $\mathrm{HD}^{+}$was probed recently ${ }^{7}$ with a fractional resolution of $3 \times 10^{-}$ 12 and a fractional uncertainty of $1.3 \times 10^{-11}$. The two-photon spectroscopy approaches are promising for the increase of the precision ${ }^{8,9}$. A two-photon rovibrational transition was also probed recently ${ }^{10}$ with a precision of $2.9 \times 10^{-12}$. This contribution proposes to characterize a THz-wave off-resonantly coupled to the rotational transition at $1.3 \mathrm{THz}$ by measurements of the lightshifts induced on a Zeeman component of the two-photon rovibrational transition at 55.9 THz. An algorithm is proposed to convert a set of scalar phase-less lightshift measurements performed for two orientations of the magnetic field in the ion trap into the amplitudes and the phases of the $\mathrm{THz}$ electric field components in the Cartesian laboratory frame.

\section{AB-INITIO CALCULATIONS OF THE HD ${ }^{+}$ION ENERGY LEVELS AND SHIFTS IN EXTERNAL FIELDS}

The rovibrational energy levels of $\mathrm{HD}^{+}$in its ground electronic state are calculated without spin effects as the sum of the solution of the nonrelativistic Schrödinger equation with a series expansion of quantum electrodynamics correction terms ${ }^{11}$. The hyperfine structure arise from the coupling of 
the proton $\vec{I}_{p}$, deuteron $\overrightarrow{\mathrm{I}}_{\mathrm{d}}$, and electron $\overrightarrow{\mathrm{S}}_{\mathrm{e}}$ spins with the rotational angular momentum $\overrightarrow{\mathrm{L}}$ that yields the total angular momentum $\overrightarrow{\mathrm{J}}: \overrightarrow{\mathrm{F}}=\overrightarrow{\mathrm{S}}_{\mathrm{e}}+\overrightarrow{\mathrm{I}}_{\mathrm{p}} ; \overrightarrow{\mathrm{S}}=\overrightarrow{\mathrm{F}}+\overrightarrow{\mathrm{I}}_{\mathrm{d}} ; \overrightarrow{\mathrm{J}}=\overrightarrow{\mathrm{L}}+\overrightarrow{\mathrm{S}}$. The hyperfine energy levels, labeled with the quantum numbers for the vibration and for the spin coupling scheme, are calculated as the eigenvalues of a Breit-type spin Hamiltonian ${ }^{12}$. A static magnetic field is used to define the quantization axis and to split the hyperfine states in magnetic subcomponents, labelled with the quantum number $J_{z}$ of the projection of $\vec{J}$ on the quantization axis. The Zeeman shift of an energy level in a static magnetic field is described with a quadratic dependence in function of the value of the magnetic field ${ }^{4}$. The total energy of a magnetic level may be expressed as :

$$
E\left(v, L, F, S, J, J_{z}\right)=E_{r v}(v, L)+E_{h f}(v, L, F, S, J)+\Delta E_{z}\left(v, L, F, S, J, J_{z} ; B\right)
$$

in function of the rovibrational energy $E_{r v}(v, L)$, the hyperfine energy $E_{h f}(v, L, F, S, J)$, and the Zeeman shift $\Delta E_{z}\left(v, L, F, S, J, J_{z} ; B\right)$, respectively.

This contribution addresses the measurement of a $\mathrm{THz}$ electric field in the Cartesian laboratory frame $\operatorname{LCF}\left(\vec{e}_{x}, \vec{e}_{y}, \vec{e}_{z}\right)$ :

$$
\overrightarrow{\mathrm{E}}(\mathrm{t})=\sum_{j=\{x, y, z\}} \frac{E_{j} \vec{e}_{j}}{2} \times \mathrm{e}^{-i\left(\omega t+\varphi_{j}\right)}+\text { c.c. }
$$

where the components are described with three real positive amplitudes $E_{j}$ and two phases $\varphi_{x}, \varphi_{y}$. Here, for simplicity, $\varphi_{z}=0$. The interaction of the THz-wave with the $\mathrm{HD}^{+}$ions may be described using the standard components of the $\mathrm{THz}$ electric field in a Cartesian reference frame with the zaxis oriented along the direction of the magnetic field $\operatorname{MICF}\left(\vec{e}_{c, x}, \vec{e}_{c, y}, \vec{e}_{c, z}\right)$ :

$$
\overrightarrow{\mathrm{E}}(\mathrm{t})=\sum_{q=\{-1,0,1\}}(-1)^{q} \frac{E_{-q} \vec{e}_{q}}{2} \times \mathrm{e}^{-i\left(\omega t+\varphi_{q}\right)}+\text { c.c. }
$$

with real positive amplitudes $E_{0}, E_{ \pm 1}$ and phases $\varphi_{0}, \varphi_{ \pm 1}$ corresponding to the linear or circular polarizations, defined by $\vec{e}_{0}=\vec{e}_{c, z}, \vec{e}_{ \pm 1}=\mp\left(\vec{e}_{c, x} \pm i \vec{e}_{c, y}\right) / \sqrt{2}$. The interaction Hamiltonian is expressed in the electric dipole approximation as :

$$
\mathrm{V}=-\vec{d} \cdot \overrightarrow{\mathrm{E}}(\mathrm{t})
$$


using the electric dipole operator $\vec{d}$ of $\mathrm{HD}^{+}$in the laboratory frame.

An energy level of $\mathrm{HD}^{+}$probed with a $\mathrm{THz}$ electric field far from the resonance with another energy level is shifted by the ac-Stark effect by an amount calculated with the second-order perturbation theory :

$$
\begin{gathered}
\delta E_{n}=-\frac{1}{4} \sum_{q=\{-1,0,1\}}(-1)^{q}\left|E_{-q}\right|^{2} \alpha_{n, q}(\omega) \\
\alpha_{n, q}(\omega)=\operatorname{Re}\left[\left\langle n\left|d_{-q} \cdot \frac{1}{\underline{\mathrm{H}}^{*}-\underline{\mathrm{E}}_{n}-\hbar \omega} \cdot d_{q}\right| n\right\rangle+\left\langle n\left|d_{-q} \cdot \frac{1}{\underline{\mathrm{H}}-\underline{\mathrm{E}}_{n}^{*}+\hbar \omega} \cdot d_{q}\right| n\right\rangle\right]
\end{gathered}
$$

The lightshift is quantified with the dynamic polarizabilities of $\mathrm{HD}^{+}$, that depend on the matrix elements of the standard components of the dipole operator $d_{-q}=\vec{d} \cdot \hat{\varepsilon}_{q}$, the unperturbed energy levels $E_{n}$, and their decay rates $\gamma_{n}$. The second line of eq. (5) introduces the standard dynamic polarizability of $\operatorname{HD}^{+} \alpha_{n, q}(\omega)$ (labeled as $\sigma^{-}, \pi, \sigma^{+}$for $\mathrm{q}=\{-1,0,1\}$ ) using the complex operator $\underline{\mathrm{H}}=H+i \hbar \Gamma$, defined such as $\left\langle n|H| n^{\prime}\right\rangle=\delta_{n n^{\prime}} E_{n}, \quad\left\langle n|\Gamma| n^{\prime}\right\rangle=\delta_{n n^{\prime}} \gamma_{n} / 2$, and its eigenvalues $\underline{\mathrm{E}}_{n}=E_{n}+i \hbar \gamma_{n} / 2$. The standard dynamic polarizabilities $\alpha_{n}\left(\left\{U_{t h}\right\} ; q, B, f_{T H z}\right)$ of the $\mathrm{HD}^{+}$energy levels are calculated in this contribution in function of a set of theoretical parameters $\left\{U_{t h}\right\}$ (the rovibrational energy levels with $10^{-12}$ fractional uncertainty ${ }^{11}$, the hyperfine splittings with $0.5 \mathrm{kHz}$ uncertainty $^{12}$, the Zeeman shift parameters with $10^{-4}$ or better fractional uncertainty ${ }^{4}$, the radiative lifetimes with ms level uncertainty ${ }^{13}$, and the electric dipole transition moments with $10^{-4}$ fractional uncertainty or better ${ }^{14}$ ), and a set of experimental parameters (polarization $q$ and frequency $f_{T H z}$ of the THz-wave, and the magnetic field strength $B$ ). The uncertainties of the polarizabilities are derived with the error propagation law, by using the uncertainties of the theoretical parameters ${ }^{11-14}$, and of the experimental parameters (the fractional uncertainty of the THz-wave frequency is assumed at $10^{-12}$, and the magnetic field uncertainty is $10^{-10} \mathrm{~T}$ as discussed in section 4).

\section{TWO-PHOTON SPECTROSCOPY SCHEME OF HD ${ }^{+}$}

The approach for THz-wave characterization proposed in this contribution (Fig. 1.a, 1.b) is based on previously developed spectroscopy experiments with cold trapped $\mathrm{HD}^{+}$ions ${ }^{7,10}$. A linear 
radiofrequency trap is exploited to trap $\sim 10^{2} \mathrm{HD}^{+}$ions and $\sim 10^{3} \mathrm{Be}^{+}$ions. Laser cooling of the $\mathrm{Be}^{+}$ ions at $313 \mathrm{~nm}$ is exploited for sympathetical cooling of $\mathrm{HD}^{+}$. An uniform static magnetic field, controlled with three coil pairs in the Helmholtz configuration which are driven with three independent current sources, is applied to the ion trap.

The measurements address the Zeeman subcomponents of the two-photon rovibrational transition $(\mathrm{v}, \mathrm{L})=(0,0) \rightarrow(2,0)$. Detection is performed by photodissociation of the $(\mathrm{v}, \mathrm{L})=(2,0)$ level with a 175 $\mathrm{nm}$ laser. The number of the $\mathrm{HD}^{+}$ions in the trap is monitored with the increase of the fluorescence of $\mathrm{Be}^{+}$ions at the excitation of the secular motion of the $\mathrm{HD}^{+}$ions in the trap. Doppler-free twophoton spectroscopy is performed with a stationary wave tuned by $55.909 \mathrm{THz}$. The time dependences of the populations in the relevant energy levels of the $\mathrm{HD}^{+}$are described by a set of rate equations that take into account the optical pumping, the transitions driven by the blackbody radiation, and the radiative relaxation. The solution allows to derive the lineshape of the two-photon resonance. The full-width half measured linewidth of the two-photon rovibrational line is $20 \mathrm{~Hz}$, by assuming a two-photon rovibrational transition rate of $10 \mathrm{~s}^{-1}$, a dissociation rate of $200 \mathrm{~s}^{-1}$ and a REMPD time of $10 \mathrm{~s}^{15}$. A THz electric field coupled off-resonantly to the rotational transition $(\mathrm{v}, \mathrm{L})=(0,0) \rightarrow(0,1)$ at $1.315 \mathrm{THz}$, or $(\mathrm{v}, \mathrm{L})=(2,0) \rightarrow(2,1)$ at $1.197 \mathrm{THz}$, respectively, is characterized using the measurements of the lightshifts induced on the transitions between the spin states with maximal $\mathrm{J}$ and maximal magnitude of the projection $\mathrm{J}_{\mathrm{Z}}= \pm \mathrm{J}$ (stretched states) of the levels $(\mathrm{v}, \mathrm{L})=(0,0)$ and $(\mathrm{v}, \mathrm{L})=(2,0)$, that have negligible sensitivity to the magnetic field.

The fractional frequency uncertainty is estimated with the expression of the Allan variance for the molecular ion quantum projection noise limit :

$$
\sigma_{y}(\tau)=\frac{1}{\pi Q \sqrt{N_{\text {ion }}}} \times \sqrt{\frac{T_{c}}{\tau}}
$$

in function of the quality factor $Q$ of the two-photon transition, the number $N_{i o n}$ of $\mathrm{HD}^{+}$ions, the cycle time $T_{c}$, and the interrogation time $\tau$. The quality factor $Q=f_{2 p h} / \Delta f_{H W H M}$, is expressed in terms of the two-photon frequency and the half-linewidth, estimated from the natural lifetimes of the $\mathrm{HD}^{+}$ ion energy levels ${ }^{13}$. Assuming the case of single ion spectroscopy experiment with $T_{c}=\tau$, the frequency uncertainty for $(\mathrm{v}, \mathrm{L})=(0,0) \rightarrow(2,0)$ line is estimated at $2.49 \mathrm{~Hz}$ and for $(\mathrm{v}, \mathrm{L})=(0,0) \rightarrow(2,2)$ line at $2.57 \mathrm{~Hz}$. 


\section{PROBING MAGNETIC FIELDS BY ZEEMAN SPECTROSCOPY OF HD ${ }^{+}$}

An alternative spectroscopic scheme aiming to characterize the magnetic field in the ion trap, is based on a sensitive Zeeman component of the rovibrational transition $(\mathrm{v}, \mathrm{L})=(0,0) \rightarrow(2,2)$ of $\mathrm{HD}^{+}$. The detection is performed similarly, by photodissociation of the $(\mathrm{v}, \mathrm{L})=(2,2)$ level with the $175 \mathrm{~nm}$ laser. Precisely, the Zeeman shift of a transition $\left(\mathrm{v}, \mathrm{L}, \mathrm{F}, \mathrm{S}, \mathrm{J}, \mathrm{J}_{\mathrm{Z}}\right)=\left(n, J_{z}\right) \rightarrow\left(n^{\prime}, J_{z}^{\prime}\right)$ may be calculated approximatively with a quadratic dependence :

$$
f_{n, n^{\prime}}(\mathrm{B})-f_{n, n^{\prime}}(\mathrm{B}=0)=\eta_{B}\left(t_{n}, q_{n}, r_{n}, t_{n^{\prime}}, q_{n^{\prime}}, r_{n^{\prime}}, J_{z}, J_{z}^{\prime}\right) B+\eta_{B^{2}}\left(t_{n}, q_{n}, r_{n}, t_{n^{\prime}}, q_{n^{\prime}}, r_{n^{\prime}}, J_{z}, J_{z}^{\prime}\right) B^{2}
$$

in function of the magnitude of the magnetic field $B$ and the linear $\eta_{B}$ and quadratic $\eta_{B^{2}}$ Zeeman shift coefficients that depend on a set of six theoretical parameters $\left\{U_{t h}\right\}=\left\{t_{n}, q_{n}, r_{n}, t_{n^{\prime}}, q_{n^{\prime}}, r_{n^{\prime}}\right\}$ that were calculated for the relevant hyperfine energy levels of $\mathrm{HD}^{+}$in ref. ${ }^{4}$.

The inversion of eq. (7) allows to derive the magnitude of the magnetic field in function of the experimental Zeeman shift, and the values for the theoretical parameters $\left\{U_{t h}\right\}$. Two values of the magnetic field may be derived from the second-order equation. The identification of the solution may be made by comparison with the value of the magnetic field measured with an accurate magnetometer. The uncertainty of the magnetic field may be calculated with the error propagation formula, in function of the contribution from the frequency shift measurement uncertainty $\sigma_{\text {exp,rv }}=2.57 \cdot \sqrt{2} \mathrm{~Hz}$, estimated with the molecular ion quantum projection noise limit, and of the contributions from the theoretical parameters, which are assumed correlated and having the same uncertainties $\sigma_{t h, q, r}=50 \mathrm{MHz} / \mathrm{T}^{2}, \sigma_{t h, t}=5 \mathrm{kHz} / \mathrm{T}$. Here, the Zeeman spectroscopy of the subcomponent $\left(\mathrm{v}, \mathrm{L}, \mathrm{F}, \mathrm{S}, \mathrm{J}, \mathrm{J}_{\mathrm{z}}\right)=(0,0,1,2,2,-2) \rightarrow(0,2,1,2,4,0)$ is addressed for measuring the magnitude of the magnetic field. The dependence of the fractional uncertainty of the magnitude of the magnetic field is plotted in Fig. 2. The sensitivity and the precision of the calibration of the magnetic field are limited by the frequency measurement uncertainty by $5 \times 10^{-10} \mathrm{~T}$. The contribution of the theoretical uncertainties to the precision of the magnetic field remains at a fractional value beyond the $10^{-6}$ level. 


\section{PROBING THZ ELECTRIC FIELDS BY LIGHTSHIFT MEASUREMENTS}

\subsection{Scalar THz electrometry by $\mathrm{HD}^{+}$spectroscopy : accuracy and sensitivity issues}

The total lightshift induced by a THz-wave on a two-photon rovibrational transition $\left(\mathrm{v}, \mathrm{L}, \mathrm{F}, \mathrm{S}, \mathrm{J}, \mathrm{J}_{\mathrm{Z}}\right)=(\mathrm{n}) \rightarrow\left(\mathrm{n}\right.$ ') may be expressed in function of the amplitude of the electric field $E_{T H z}$ of the THz-wave:

$$
f_{n \rightarrow n^{\prime}}\left(E_{T H z}\right)-f_{n \rightarrow n^{\prime}}\left(E_{T H z}=0\right)=-\frac{\left|E_{T H z}\right|^{2}}{8}\left(\alpha_{n^{\prime}}\left(\left\{U_{t h}\right\} ; B, f_{T H z}\right)-\alpha_{n}\left(\left\{U_{t h}\right\} ; B, f_{T H z}\right)\right)
$$

The $\mathrm{HD}^{+}$frequency measurements may be converted into measurements of the THz-wave electric field by using the total dynamic polarizabilities of the $\mathrm{HD}^{+}$energy levels that are calculated theoretically. For the propagation of a plane electromagnetic wave, assumed here, the electric field is related to the intensity of the THz-wave as : $I_{T H z}=c \varepsilon_{0} E_{T H z}^{2}$, in function of the speed of the light c, and the vacuum permittivity $\varepsilon_{0}$.

The sources of uncertainty of the THz-wave intensity are the frequency measurement errors that translate into uncertainties of the lightshift and of the magnetic field magnitude, and uncertainties of the theoretical parameters that contribute to the uncertainty of the dynamic polarizability and of the magnetic field magnitude. The uncertainty of the THz-wave intensity is estimated with the error propagation formula :

$$
u\left(I_{T H z}\right)=\sqrt{\left(\frac{\left(8 c \varepsilon_{0}\right) \times u(\delta f)}{\Delta \alpha\left(\left\{U_{t h}\right\} ; B, f_{T H z}\right)}\right)^{2}+\left(\frac{I_{T H z} u\left(\Delta \alpha\left(\left\{U_{t h}\right\} ; B, f_{T H z}\right)\right)}{\Delta \alpha\left(\left\{U_{t h}\right\} ; B, f_{T H z}\right)}\right)^{2}}
$$

in function of the lightshift measurement uncertainty $u(\delta f)$, and the uncertainty of the dynamic polarizability $u\left(\Delta \alpha\left(\left\{U_{t h}\right\} ; B, f_{T H z}\right)\right)$. The first term on the right side of the eq. (10) gives the sensitivity limit for the detection of the THz-wave by lightshift measurements using a two-photon transition. In order to minimize the calibration uncertainty for a given intensity, polarization and frequency of the THz-wave, the two-photon transition should have the highest dynamic polarizability, the lightshift measurements should be performed with the lowest uncertainties, and the dynamic polarizability should be estimated with the lowest uncertainty. Particularly, the choice of the magnitude of the magnetic field can optimize the value and the uncertainty of the dynamic polarizability. 
In the case of the detection of a THz-wave at $1314947502.3 \mathrm{kHz}$ with $\pi$ polarization by using lightshift measurements on the $\left(\mathrm{v}, \mathrm{L}, \mathrm{F}, \mathrm{S}, \mathrm{J}, \mathrm{J}_{\mathrm{Z}}\right)=(0,0,1,2,2,2) \rightarrow(2,0,1,2,2,2)$ two-photon transition with an experimental uncertainty of $u(\delta f)=2.49 \cdot \sqrt{2} \mathrm{~Hz}$, the optimization procedure for the magnetic field magnitude yields the smallest experimental uncertainty of the THz-wave intensity at $4.4 \times 10^{-8}$ $\mathrm{W} / \mathrm{m}^{2}$. That indicates the limit of sensitivity with the method proposed in this contribution, that is a $\mathrm{THz}$ electric field with an amplitude as low as $5.8 \times 10^{-6} \mathrm{~V} / \mathrm{m}$, which is an order of magnitude smaller than the lowest microwave electric field detected with the Rydberg atom spectroscopy $\operatorname{method}^{1}$.

The precision of the calibration of the THz-wave intensity using lightshift measurements is estimated for an optimized value of the magnetic field in the range $0-10^{-4} \mathrm{~T}$ that minimizes the total uncertainty estimated with eq. (10), and plotted in Fig. 3. For THz-wave intensities higher than 0.1 $\mathrm{W} / \mathrm{m}^{2}$ the optimized value for the magnetic field is negative and therefore fixed at 0 . The best fractional uncertainty is at the $10^{-3}$ level. The contribution to the fractional uncertainty arising solely from the frequency measurement errors is by an order of magnitude smaller than the total fractional uncertainty or better. For a THz-wave intensity of $1 \mathrm{~W} / \mathrm{m}^{2}$, the fractional uncertainty is limited by the accuracy of the dynamic polarizability calculation at the $10^{-2}$ level, while the experimental fractional uncertainty is at the $10^{-4}$ level. The limit of sensitivity of this method may be extrapolated in this case at the $10^{-7} \mathrm{~W} / \mathrm{m}^{2}$ level.

\subsection{Retrieval of the polarization ellipse of a THz-wave by $\mathrm{HD}^{+}$spectroscopy}

The THz electric field vector is described in eq. (2) with three linearly polarized components along each axis of a fixed Cartesian laboratory coordinate frame $\operatorname{LCF}\left(\vec{e}_{x}, \vec{e}_{y}, \vec{e}_{z}\right)$. The axes of the laboratory frame are aligned following the orientations of the coil pairs, which are supposed orthogonal. Alternatively, the $\mathrm{THz}$ electric field may be decomposed in standard components in $\operatorname{MICF}\left(\vec{e}_{c, x}, \vec{e}_{c, y}, \vec{e}_{c, z}\right)$, as indicated in eq. (3). The orientation of MICF relative to $L C F$, defined with the Euler angles $(\alpha, \beta, \zeta=0)$, may be changed by varying the currents in the coils. Each standard component of the THz electric field, denoted with $E_{\pi, \sigma^{ \pm}}^{(\alpha, \beta)}$, addresses off-resonantly $\pi$ or $\sigma^{ \pm}$Zeeman subcomponents of the $(\mathrm{v}, \mathrm{L})=(\mathrm{v}, 0) \rightarrow(\mathrm{v}, 1)$ transitions and induces a lightshift in proportion with its 
squared amplitude. The squared modules of the standard components may be related to the $\mathrm{THz}$ electric field amplitudes and phases in the laboratory frame $\left(E_{x}, E_{y}, E_{z}, \varphi_{x}, \varphi_{y}\right)^{16}$.

For a given orientation of the magnetic field, three independent measurements of lightshifts $\delta f_{k}^{(\alpha, \beta)}, k=\{1,2,3\}$ allow to derive the standard components of the $\mathrm{THz}$ electric field, by solving a nonsingular system with three equations with the appropriate differential standard dynamic polarizabilities. Possible approaches that may provide independent lightshift measurements are to use three different values of the magnetic field, or to address three different Zeeman or hyperfine subcomponents of the two-photon transition. In order to determine all parameters of the $\mathrm{THz}$ electric field in the laboratory frame, it is necessary also to change the orientation of the magnetic field. The choice of orientations of the magnetic field is linked to the periodicity of the transformation from the standard components of the $\mathrm{THz}$ electric field in the rotated frame to the Cartesian components in the laboratory frame. At least five measurements of the standard components $E_{\pi}^{(\alpha, \beta)}, E_{\sigma^{*}}^{(\alpha, \beta)}$ at two different orientations $(\alpha, \beta)$ of the magnetic field are required. For example, one can exploit the measurement of the set of amplitudes $\left(E_{\pi}^{(0,0)}, E_{\sigma^{+}}^{(0,0)}, E_{\sigma^{-}}^{(0,0)}, E_{\sigma^{+}}^{(0, \pi / 2)}, E_{\sigma^{-}}^{(0, \pi / 2)}\right)$ to derive analytically the parameters of the $\mathrm{THz}$ electric field in the laboratory frame ${ }^{16}$.

The measurement of a reference THz-wave oscillating at $f_{T H z}=1314947502.3 \mathrm{kHz}$ with the following parameters of the electric field in the laboratory frame $\left(E_{x}=E_{y}=E_{z}=15.83 \mathrm{mV} / \mathrm{m}, \varphi_{x}=\pi / 4, \varphi_{y}=\pi / 3\right)$ is presented here. The measurement addresses the lightshifts of the two-photon transition $\left(\mathrm{v}, \mathrm{L}, \mathrm{F}, \mathrm{S}, \mathrm{J}, \mathrm{J}_{\mathrm{Z}}\right)=(0,0,1,2,2,2) \rightarrow(2,0,1,2,2,2)$ for two orientations of the magnetic field $(\alpha, \beta)=(0,0),(\alpha, \beta)=(0, \pi / 2)$ and three values of the magnitude of the magnetic field $B_{1}=10^{-6} \mathrm{~T}, B_{2}=5 \times 10^{-6} \mathrm{~T}, B_{3}=10^{-5} \mathrm{~T}$. The six experimental lightshifts are estimated by adding the lightshifts calculated for the reference THz-wave with random error values described with a Gaussian distribution with zero mean and covariance equal to the squared uncertainty given by the molecular ion quantum projection noise limit for the frequency measurements. The standard components of the $\mathrm{THz}$ electric field are subsequently derived using the relevant set of eighteen differential standard dynamic polarizabilities. The polarizabilities are estimated as the theoretical values plus a random contribution with a Gaussian distribution with zero mean and variance equal to the squared uncertainty of each polarizability calculation. From the calculated values of the standard 
components, the parameters of the $\mathrm{THz}$ electric field in the laboratory frame are determined analytically. The retrieved polarization ellipses of the THz electric field are represented on Fig. 4 for 10 simulated sets of lightshifts. The values and the uncertainties of the parameters of the THz-wave determined from a set of 100 simulations of lightshifts are:

$$
\begin{gathered}
E_{x}=15.88(92) \mathrm{mV} / \mathrm{m}, E_{y}=15.74(72) \mathrm{mV} / \mathrm{m}, E_{z}=15.831(3) \mathrm{mV} / \mathrm{m} \\
\varphi_{x}=0.78(6), \varphi_{y}=1.05(1)
\end{gathered}
$$

The accuracy of the calibration is better than $5 \%$ for the components of the electric field and better than $10 \%$ for the phases. The shift between the initially assumed value and the determined value for each parameter of the THz-wave is consistent with the calculated uncertainty.

\section{CONCLUSION}

This contribution proposes the characterization of the electromagnetic fields from the comparison of the measurements of the frequency shifts induced on two-photon transitions of cold trapped HD ions with the predictions of the molecular ion theory. The Zeeman shift for a selected hyperfine component of a two-photon rovibrational transition may be exploited for the measurement of the magnitude of a static magnetic field with an uncertainty at the $10^{-10} \mathrm{~T}$ level. The amplitude of the electric field of a THz-wave may be determined by the lightshift induced on a selected Zeeeman component of a two-photon transition. The sensitivity of the precision spectroscopy-based method is pushed here at the $10^{-6} \mathrm{~V} / \mathrm{m}$ level. A THz-wave with an intensity of $1 \mathrm{~W} / \mathrm{m}^{2}$ can be calibrated with an accuracy limited at the $10^{-2}$ level by the accuracy of the theoretical calculations, and at the $10^{-4}$ level by the experimental errors. In addition, the polarisation ellipse of a THz-wave may be retrieved from the measurements of the lightshifts induced on a Zeeman component of a two-photon transition for different orientations of the magnetic field in the ion trap. The response of $\mathrm{HD}^{+}$to the external field obtained with this method may be referenced directly to a frequency standard, that is advantageous comparing to similar approaches based on the Rabi-rate measurements with the Rydberg atoms ${ }^{16,17}$. The precision of the calibration of a reference THz-wave using a set of lightshift measurements is estimated better than $5 \%$ for the electric field components and better than $10 \%$ for the phases. 


\section{REFERENCES}

[1] Kumar, S. et al, "Rydberg-atom based radio-frequency electrometry using frequency modulation spectroscopy in room temperature vapour cells," Opt. Express 25 (8), 8625-8637 (2017). [2] Korobov, V.I., Hilico, L. and Karr, J.-Ph., "Fundamental transitions and ionization energies of the hydrogen molecular ions with few ppt uncertainty," Phys. Rev. Lett. 118 (23), 233001 (2017). [3] Korobov, V.I., Karr, J.-Ph., Haidar, M. and Zhong, Z.-X., "Hyperfine structure in the $\mathrm{H}_{2}{ }^{+}$and $\mathrm{HD}^{+}$molecular ions at order ma $\alpha^{6}$, Phys. Rev. A 102 (2), 022804 (2020).

[4] Bakalov, D., Korobov, V.I. and Schiller, S., "Magnetic field effects in the transitions of the $\mathrm{HD}^{+}$molecular ion and precision spectroscopy,” J. Phys. B 44 (2), 025003 (2011).

[5] Schiller, S., Bakalov, D., Bekbaev, A.K. and Korobov, V.I., "Static and dynamic polarizability and the Stark and blackbody-radiation frequency shifts of the molecular hydrogen ions $\mathrm{H}_{2}^{+}, \mathrm{HD}^{+}$, and $\mathrm{D}_{2}^{+}$," Phys. Rev. A 89 (5), 052521 (2014).

[6] Bakalov, D. and Schiller, S., "The electric quadrupole moment of molecular hydrogen ions and their potential for a molecular ion clock,” Appl. Phys. B 114, 213-230 (2014).

[7] Alighanbari, S., Giri, G.S., Constantin, F.L., Korobov, V. and Schiller, S., "Precise test of quantum electrodynamics and determination of fundamental constants with $\mathrm{HD}^{+}$ions," Nature 581, $152-158$ (2020).

[8] Tran, V.Q., Karr, J.-Ph., Douillet, A., Koelemeij, J.C.J., Hilico, L., "Two-photon spectroscopy of trapped $\mathrm{HD}^{+}$ions in the Lamb-Dicke regime,” Phys. Rev. A 88 (3), 033421 (2013).

[9] Constantin, F.L., "THz/infrared double resonance two-photon spectroscopy of $\mathrm{HD}^{+}$for determination of fundamental constants," Atoms 5 (4), 38 (2017).

[10] Patra, S. et al, "Proton-electron mass ratio from laser spectroscopy of $\mathrm{HD}^{+}$at the part-pertrillion level," Science 369 (6508), 1238-1241 (2020).

[11] Moss, R.E., "Calculations for vibration-rotation levels of $\mathrm{HD}^{+}$, in particular for high N," Mol. Phys. 78 (2), 371-405 (1993).

[12] Bakalov, D., Korobov, V.I. and Schiller, S., "High-precision calculation of the hyperfine structure of the HD' ion," Phys. Rev. Lett. 97 (24), 243001 (2006).

[13] Amitay, Z., Zajfman, D. and Forck, P., "Rotational and vibrational lifetime of isotopically asymmetrized homonuclear diatomic molecular ions," Phys. Rev. A 50 (3), 2304-2308 (1994). 
[14] Bakalov, D. and Schiller S., "Static Stark effect in the molecular ion $\mathrm{HD}^{+}$," Hyperfine Interact. 210 (1-3), 25-31 (2012).

[15] Constantin, F.L., "Double-resonance two-photon spectroscopy of hydrogen molecular ions for improved determination of fundamental constants," IEEE Trans. Instrum. Meas. 68 (6), 21512159 (2019).

[16] Koepsell, J., Thiele, T., Deiglmayr, J., Wallraff, A. and Merkt, F., "Measuring the polarisation of electromagnetic fields using Rabi-rate measurements with spatial resolution : Experiment and theory," Phys. Rev. A 95 (5), 053860 (2017).

[17] Thiele, T., Lin, Y., Brown, M.O. and Regal, C.A., "Self-calibrating vector atomic magnetometry through microwave polarization reconstruction,” Phys. Rev. Lett. 121 (10), 153202 (2018). 


\section{Figure captions :}

Figure 1. a. The experimental setup and the reference coordinate frames. The THz-wave (blue line) is off-resonantly coupled to the energy levels of the $\mathrm{HD}^{+}$ions embedded in a Coulomb crystal. Twophoton spectroscopy is performed with a stationary wave from the IR laser (red line). $\mathrm{Be}^{+}$ions are cooled with the $313 \mathrm{~nm}$ laser (magenta line). The detection is performed by dissociating $\mathrm{HD}^{+}$ions with the $175 \mathrm{~nm}$ laser (green line). The static magnetic field in the ion trap (olive line) can be oriented to any direction, defined with the Euler angles $(\alpha, \beta)$ in the Cartesian Laboratory Coordinate Frame $(\mathrm{x}, \mathrm{y}, \mathrm{z})$ (black lines). The standard components of the THz electric field are addressed in the Cartesian Molecular Ion Coordinate Frame $\left(\mathrm{x}_{\mathrm{c}}, \mathrm{y}_{\mathrm{c}}, \mathrm{z}_{\mathrm{c}}\right)$ (black lines). b. Rotation-vibration energy levels of $\mathrm{HD}^{+}$addressed in the $\mathrm{THz}$ sensing scheme. The two-photon rovibrational transitions are shown with red lines with arrows, the dissociation with green lines with arrows, the THz-wavedriven electric dipole couplings with blue lines with arrows and the blackbody-radiation-driven transitions with black lines and curves with arrows.

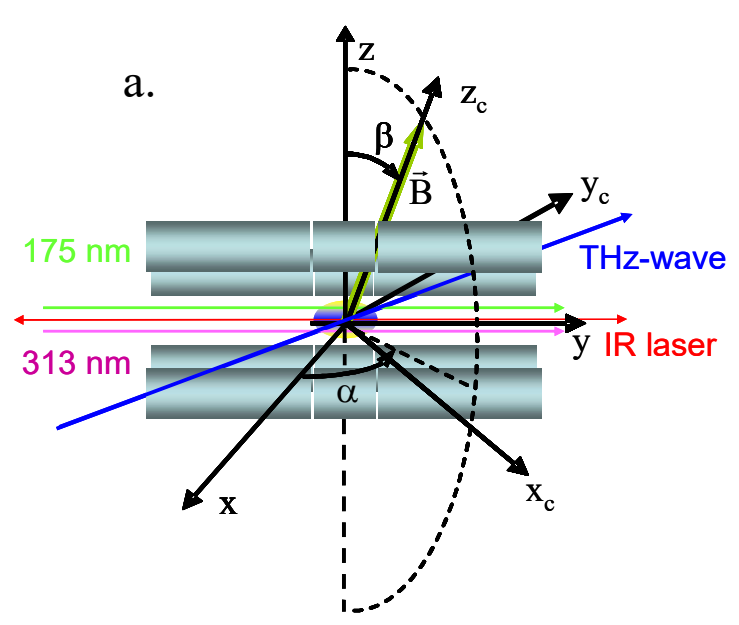

b.

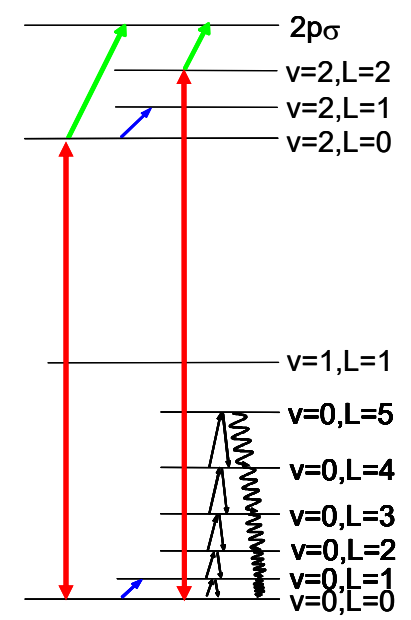


Figure 2. The relative uncertainty of the magnitude of the magnetic field determined by Zeeman spectroscopy of the two-photon transition $\left(\mathrm{v}, \mathrm{L}, \mathrm{F}, \mathrm{S}, \mathrm{J}, \mathrm{J}_{\mathrm{Z}}\right)=(0,0,1,2,2,-2) \rightarrow(2,2,1,2,4,0)$ of $\mathrm{HD}^{+}$. The contribution from theoretical and experimental uncertainties is shown with the red line. The contribution from theoretical uncertainties is shown with the blue line.

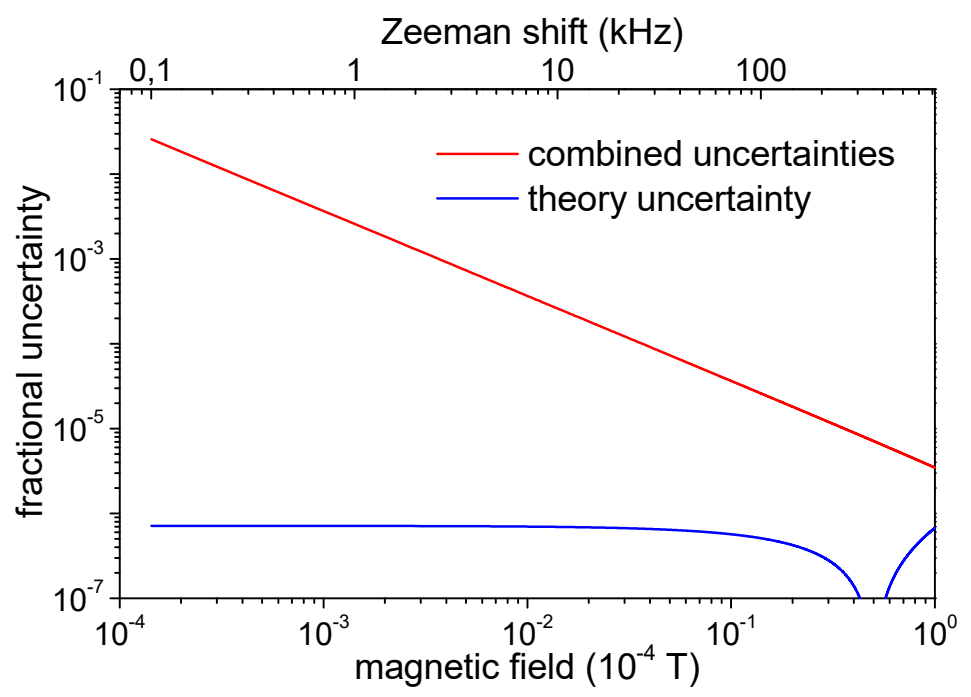


Figure 3. Dependence of the fractional uncertainty for the THz-wave electric field calibration (calculation with combined experimental and theory errors in black squares, calculation for experimental errors only in red circles) using measurements of the lightshifts of the $\left(\mathrm{v}, \mathrm{L}, \mathrm{F}, \mathrm{S}, \mathrm{J}, \mathrm{J}_{\mathrm{Z}}\right)=(0,0,1,2,2,2) \rightarrow(2,0,1,2,2,2)$ two-photon transition of $\mathrm{HD}^{+}$. The magnetic field value is optimized to minimize the total uncertainty. The THz-wave frequency is 1314947502.3 $\mathrm{kHz}$ and the polarization $\sigma^{-}$.

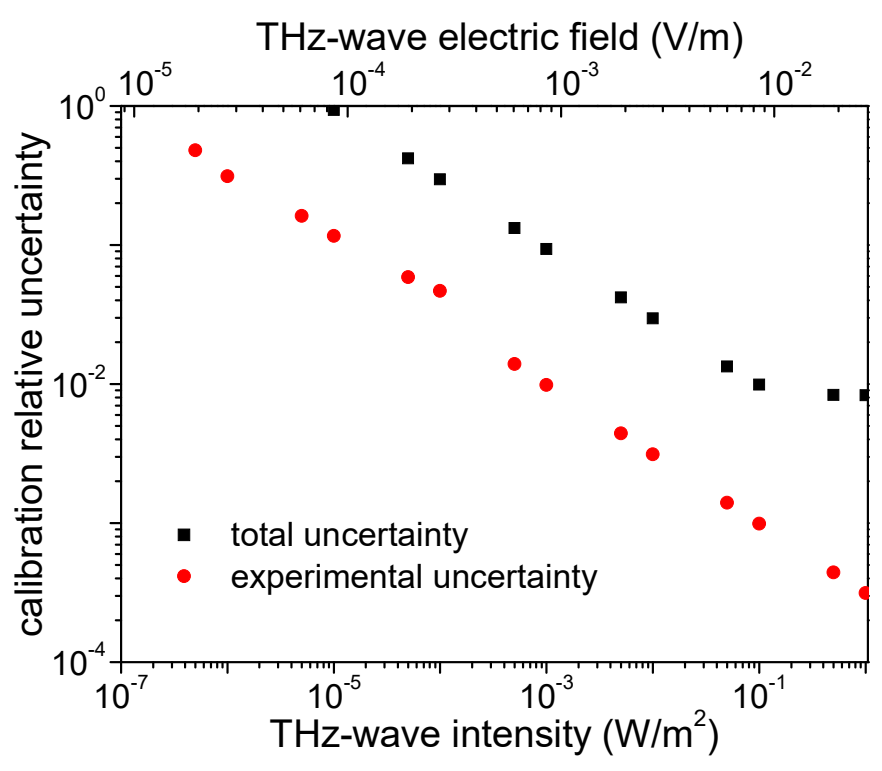


Figure 4. Polarization ellipse of a reference THz-wave (red curve). Retrieved polarization ellipses of the electric field of the THz-wave using the measurements of the lightshifts of the two-photon transition $\left(\mathrm{v}, \mathrm{L}, \mathrm{F}, \mathrm{S}, \mathrm{J}, \mathrm{J}_{\mathrm{Z}}\right)=(0,0,1,2,2,2) \rightarrow(2,0,1,2,2,2)$.

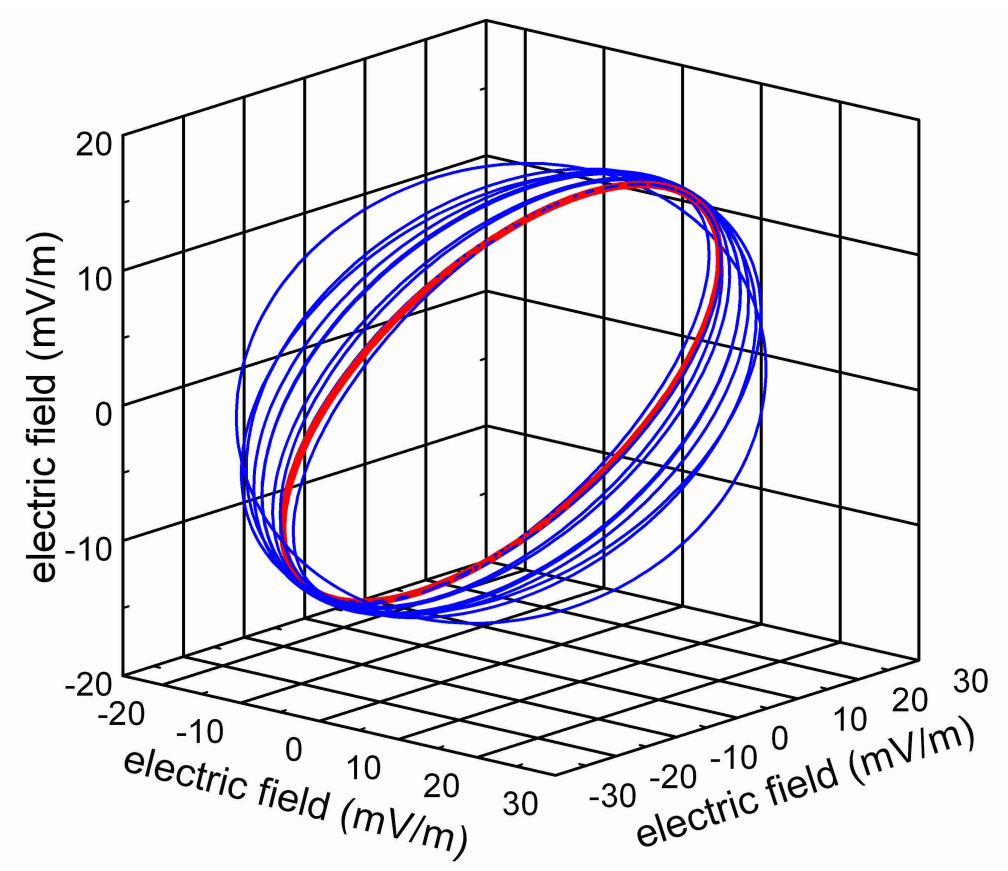

